\title{
Myocardial Infarction caused by Triple-Hit Lymphoma
}

\section{Amanuel $\mathbf{H}^{1^{*}}$, Schofield $\mathrm{J}^{2}$ and Kotoucek PP}

${ }^{1}$ Department of Haematology, Darent Valley Hospital, United Kingdom

${ }^{2}$ Department of Histopathology, Maidstone Hospital, United Kingdom

*Corresponding author: Amanuel H, Department of Haematology, Darent Valley Hospital, United Kingdom, Tel: 00447506046910, E-mail: hermonamanuel@doctors.org.uk

Received date: February 12, 2018; Accepted date: April 18, 2018; Published date: April 25, 2018

Copyright: ( 2018 Amanuel $\mathrm{H}$, et al. This is an open-access article distributed under the terms of the Creative Commons Attribution License, which permits unrestricted use, distribution, and reproduction in any medium, provided the original author and source are credited.

\begin{abstract}
Triple Hit Lymphoma (THL) is an extremely rare and aggressive form of Non-Hodgkin's lymphoma with morphologic, phenotypic and genetic features of both diffuse large B cell lymphoma (DLBCL) and Burkitt's lymphoma (BL). Its characteristic cytogenetic abnormalities involve chromosomal rearrangements of c-MYC, BCL-2, and BCL- 6 genes. It has been recognised, in the 2016-revised WHO classification of lymphoid neoplasms, as "Highgrade B-cell lymphoma, with MYC and BCL2 and/or BCL6 rearrangements". We describe a case of a 68 years old male with two years history of stable low-grade follicular lymphoma suddenly transforming into acute leukaemia caused by THL. During the aggressively progressive phase, he developed Non-ST Elevation MI (NSTEMI), diagnosed by raised troponin and new anterolateral ST depressions on his ECG. His MI was attributed to leukostasis, anaemia and coagulopathy. THL carries poorer prognosis than either DLBCL or BL alone; thus it should be recognised as haematological emergency.
\end{abstract}

Keywords: Triple Hit Lymphoma (THL); Acute leukaemia; NON-ST elevation myocardial infarction; Haematology emergency

\section{Introduction}

Lymphomas, malignant neoplasms of the lymphocyte cell lines, are divided into two major categories, Hodgkin's lymphoma (HL) and non-Hodgkin's lymphoma (NHL). NHL is by far the most common and has myriad of subtypes such as follicular lymphoma (FL) and Diffuse large B-cell lymphoma (DLBCL) [1].

Triple Hit Lymphoma (THL) is a new extremely rare and aggressive form of NHL. Its characteristic cytogenetic abnormalities involve chromosomal rearrangements of c-MYC, BCL-2, and BCL-6 genes. It is now defined, in the 2016-revised WHO classification of lymphoid neoplasms, as High-grade B-cell lymphoma, with MYC, BCL2 and/or BCL6 translocations [2].

Morphologic, phenotypic, and genetic features intermediate between diffuse large B-cell lymphoma (DLBCL) and Burkitt's lymphoma (BL) [3]. It is extremely aggressive with well-documented leukaemic presentations [4]. The acute and aggressive transformation makes this diagnosis as a haematology emergency [5], hence early diagnosis and treatment is crucial in preventing life threatening complications. This case report describes an unfortunate and dramatic transformation of a quiescent follicular lymphoma into the most lethal form of haematological malignancy, THL, in a short period of time. THL exhibit clinically very aggressive behaviour with worse prognosis than DLBCL and/or BL [3]. This case also describes acute myocardial infraction as a complication of the acute leukaemic transformation and the clinical challenges we faced in managing him.

\section{Case Report}

$\mathrm{Mr} \mathrm{MH}$, a 68 year old male was diagnosed with grade 1 follicular lymphoma in May 2014 and was under watchful-waiting. He had a background history of mild hypertension and Gleason $3+3$ adenocarcinoma of prostate under active surveillance. He was otherwise fit and well and was non-smoker.

He was recently admitted under the surgical team with two weeks history of rectal bleed, diarrhoea and left iliac fossa pain. Admission full blood count showed mild anaemia (haemoglobin $128 \mathrm{~g} / \mathrm{l}$ ), thrombocytopaenia (platelet count $78 \times 10^{9} \mathrm{~g} / \mathrm{l}$ ), and mild leucocytosis (white blood cell count $20.3 \times 10^{9} \mathrm{~g} / 1$ with neutrophilia) (Table 1 ).

Staging CT ruled out diverticulitis but showed splenomegaly measuring $18.5 \mathrm{~cm}$ and widespread abdominal lymphadenopathy, particularly in the left external iliac chain, the largest of which measuring $4.4 \mathrm{~cm}$.

\begin{tabular}{|c|c|c|c|c|c|c|c|c|c|c|c|}
\hline & \multicolumn{3}{|c|}{ Last Blood test } & \multicolumn{3}{|c|}{ NSTEMI day } & \multicolumn{2}{|l|}{ Recall day } & \multirow{2}{*}{$\begin{array}{l}\text { D/C day } \\
22 / 10 / 2015 \\
(05: 46)\end{array}$} & \multirow{2}{*}{$\begin{array}{l}\begin{array}{l}\text { Day 1 R- } \\
\text { CHOP }\end{array} \\
\begin{array}{l}17 / 10 / 15 \\
(02: 19)\end{array}\end{array}$} & \multirow{2}{*}{$\begin{array}{l}\begin{array}{l}\text { Initial } \\
\text { Admission }\end{array} \\
02 / 10 / 15 \\
(10: 35)\end{array}$} \\
\hline & $\begin{array}{l}09 / 11 / 15 \\
(05: 43)\end{array}$ & $\begin{array}{l}07 / 11 / 2015 \\
(9: 54)\end{array}$ & $\begin{array}{l}06 / 11 / 15 \\
(10: 57)\end{array}$ & $\begin{array}{l}03 / 11 / 15 \\
(05: 04)\end{array}$ & $\begin{array}{l}02 / 11 / 2015 \\
(11: 40)\end{array}$ & $\begin{array}{l}31 / 10 / 15 \\
(05: 00)\end{array}$ & $\begin{array}{l}30 / 10 / 2015 \\
(17: 45)\end{array}$ & $\begin{array}{l}27 / 10 / 2015 \\
(12: 06)\end{array}$ & & & \\
\hline Blasts & - & - & 217.2 & - & 5.9 & 6.6 & - & - & - & - & 3.2 \\
\hline
\end{tabular}


Citation: $\quad$ Amanuel H, Schofield J, Kotoucek PP (2018) Myocardial Infarction caused by Triple-Hit Lymphoma. J Blood Disord Transfus 9: 399.

Page 2 of 3

\begin{tabular}{|l|l|l|l|l|l|l|l|l|l|l|l|}
\hline $\begin{array}{l}\text { Haemogl } \\
\text { obin }\end{array}$ & $85.0 \leftarrow$ & $94.0 \leftarrow$ & $95.0 \leftarrow$ & $75.0 \leftarrow$ & $83.0 \leftarrow$ & $88.0 \leftarrow$ & $94.0 \leftarrow$ & $88.0 \leftarrow$ & $85.0 \leftarrow$ & $79.0 \leftarrow$ & $128.0 \leftarrow$ \\
\hline $\begin{array}{l}\text { Haemato } \\
\text { crit }\end{array}$ & $0.26 \leftarrow$ & $0.28 \leftarrow$ & $0.28 \leftarrow$ & $0.22 \leftarrow$ & $0.24 \leftarrow$ & $0.26 \leftarrow$ & $0.28 \leftarrow$ & $0.26 \leftarrow$ & $0.25 \leftarrow$ & $0.23 \leftarrow$ & 0.38 \\
\hline $\begin{array}{l}\text { Lymph } \\
\text { count }\end{array}$ & $21.1 \rightarrow$ & $14.6 \rightarrow$ & - & $4.3 \rightarrow$ & - & - & 1.9 & $0.4 \leftarrow$ & $0.2 \leftarrow$ & 1.1 & - \\
\hline MCV & 87.2 & 86.4 & 86 & 84.1 & 83.3 & 82.1 & 83.5 & 85 & 86.1 & 83.5 & 85.1 \\
\hline $\begin{array}{l}\text { Neut } \\
\text { Count }\end{array}$ & $295.0 \rightarrow$ & $251.3 \rightarrow$ & - & $20.4 \rightarrow$ & - & - & $23.3 \rightarrow$ & 3.2 & $0.6 \leftarrow$ & $1.4 \leftarrow$ & - \\
\hline $\begin{array}{l}\text { Neutroph } \\
\text { ils }\end{array}$ & - & - & 6.8 & - & $34.9 \rightarrow$ & $14.1 \rightarrow$ & - & - & - & - & 7.7 \\
\hline $\begin{array}{l}\text { Platelet } \\
\text { Count }\end{array}$ & $55 \leftarrow$ & $85 \leftarrow$ & $37 \leftarrow$ & $85 \leftarrow$ & $88 \leftarrow$ & $102 \leftarrow$ & $107 \leftarrow$ & $26 \leftarrow$ & $16 \leftarrow$ & $13 \leftarrow$ & $78 \leftarrow$ \\
\hline WBC & $332.7 \rightarrow$ & $275.9 \rightarrow$ & $226.3 \rightarrow$ & $85.3 \rightarrow$ & $65.8 \rightarrow$ & $32.8 \rightarrow$ & $29.8 \rightarrow$ & $3.8 \leftarrow$ & $0.8 \leftarrow$ & $2.6 \leftarrow$ & $20.3 \rightarrow$ \\
\hline
\end{tabular}

Table 1: Blood test results showing progression of disease at the relevant stages.

Lymph node biopsy of the left external iliac chain confirmed Grade 3A follicular lymphoma. Subsequent bone marrow biopsy showed $87 \%$ infiltration by acute lymphoblastic leukaemia with rearrangement of all MYC, BCL2 and BCL6 by Fluorescence In Situ Hybridisation (FISH) (Figures 1-3). Subsequently an invasive therapy of Rituximab, Cyclophosphamide, Doxorubicin, Vincristine and Prednisolone (RCHOP) chemotherapy was commenced. Despite chemotherapy induced tumour lysis syndrome patient recovered well and completed the first cycle of chemotherapy successfully. He was discharged on day 20 of admission.

On day 8 post discharge, the patient was readmitted with a fully blown acute leukaemia likely due to selecting resistant clones under pressure of chemotherapy (Table 1). In the subsequent few days, his cell count tripled. On day 4 of his readmission he developed severe dull central chest pain radiating down his left arm. On examination he was in distress, clammy and tachycardic. Observations showed BP 80/55 mmHg, HR $126 \mathrm{bpm}$, RR 17, Sats $100 \%$ on 2 litres of oxygen via nasal prongs and Apyrexial. Initial ECG showed Anterolateral ST depression and narrow complex tachycardia (Figure 4). His first troponin-I was $874 \mathrm{ng} / \mathrm{L}$ (normal range 0-40 ng/L), Creatinine $137 \mathrm{mmol} / \mathrm{l}$, Urea 2.3 $\mathrm{mmol} / \mathrm{l}$, eGFR $45 \mathrm{ml} / \mathrm{min}$, Potassium $3.7 \mathrm{mmol} / \mathrm{l}$, Sodium $129 \mathrm{mmol} / \mathrm{l}$ and Magnesium $0.65 \mathrm{mmol} / \mathrm{l}$. A repeat 12-hour troponin-I was 5520 $\mathrm{ng} / \mathrm{L}$ (Table 1) serial troponins were not indicated as deemed unlikely to alter management. ECG abnormalities resolved slowly over the following 24 hours. Repeat ECG the following day showed sinus rhythm with T-wave inversions on lead III and aVF. He was diagnosed as Non-ST Elevation Myocardial Infarction (NSTEMI). In view of his thrombocytopaenia and anaemia he was not suitable for Aspirin, Clopidogrel and Fondaparinux. He was started on Dalteparin 5000 unit and blood and platelet support. Subsequent echocardiogram did not show any structural heart disease. His MI was attributed to leukostasis and anaemia. Mr MH died within 6 weeks of his initial hospital admission. His death was thought to be multifactorial, bone marrow failure due to disease progression, electrolyte disturbance and MI, although post mortem examination was not held.

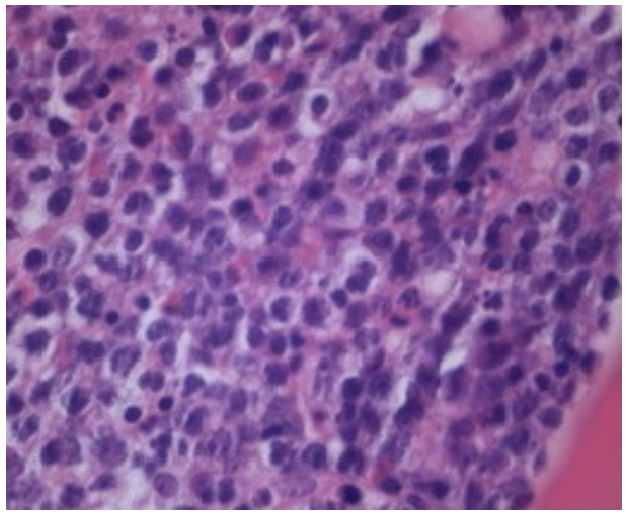

Figure 1: A high power H\&E of the bone marrow biopsy.

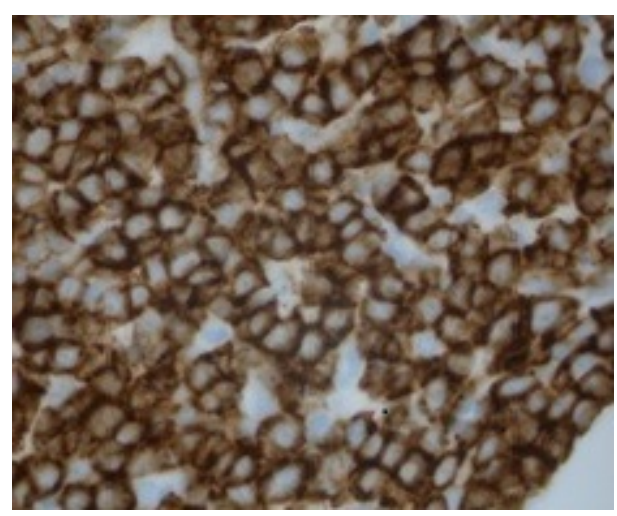

Figure 2: A high power CD20 of the bone marrow biopsy. 


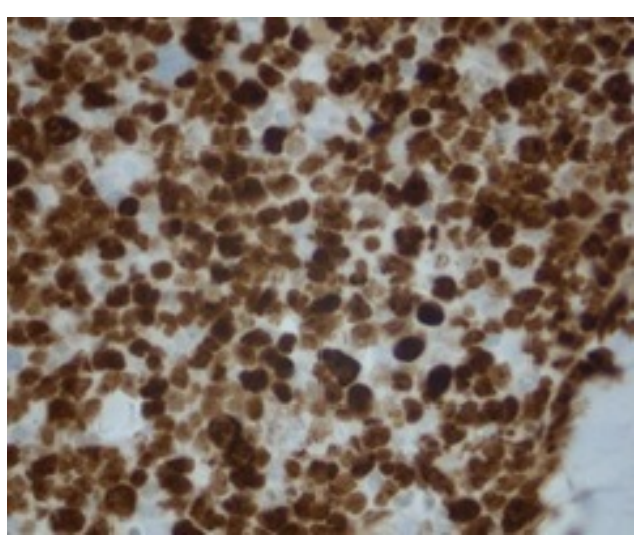

Figure 3: A high power Ki67/MIB1 of the bone marrow biopsy.

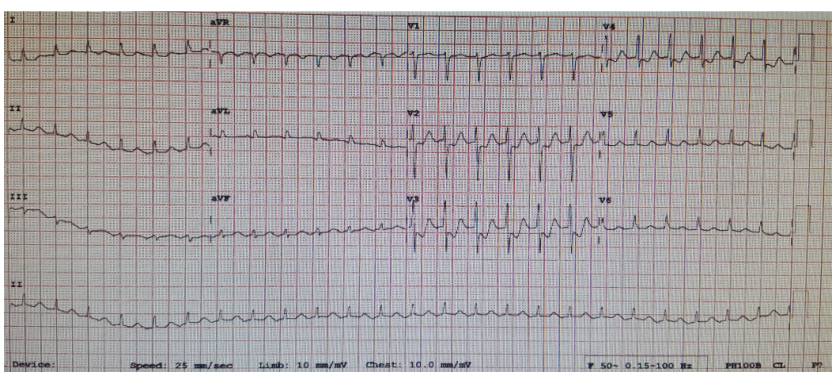

Figure 4: ST depression on V2, V3, V4 and Lead I with Sinus tachycardia.

\section{Discussion}

Lymphomas with MYC, BCL2, and BCL6 re-arrangement/ translocations, so-called THL are fairly new entity in the realm of haematologic malignancies. These are now known to be among the most aggressive of lymphomas with a rapid rate of progression, multisystem involvement and poor response to treatment. There are a few case reports and small case series that have been published; however the prevalence and incidence is not yet elucidated [6]. The prognosis post R-CHOP chemotherapy in THL is extremely poor especially when unexpected complications occur, as in our case report. There is no standardised therapy available but early allogeneic bone marrow transplant offers better hope.

Myocardial infraction is fairly common occurrence in Lymphoproliferative and myeloproliferative disorders [7]. However, its management poses significant clinical dilemma. The management of acute myocardial infarction normally involves an early administration of dual antiplatelet agents, an anticoagulant and early angioplasty $[8,9]$. Coronary artery thrombus is believed to be rich in platelets and fibrin, thus maximal inhibition of each component can prevent further clot propagation and improve prognosis [8]. Antithrombotic therapies were contraindicated in our case in view of the increased risk of bleeding from severe thrombocytopaenia, platelet dysfunction, and systemic coagulopathy. There were no reported cases of myocardial infarction in THL, to our knowledge, and there was no clear guidance in the management of such cases. Thus managing our patient proved very difficult.

\section{Conclusion}

We present this case to share lessons we have learned, which are:

- Triple hit lymphoma is a rare but very aggressive entity and patients with this disease should be managed on haematology high dependency units as a haematologic emergency.

- Early screening of all high grade lymphomas for all three gene rearrangements, c-MYC, BCL2, BCL6, is essential.

- Once THL is confirmed, early frank discussion with patient and family about the extremely poor prognosis, early palliative care involvement and early referral for allogeneic bone marrow transplant are necessary.

- The acute leucocytosis, anaemia and electrolyte disturbance secondary to disease progression can lead to acute myocardial infraction thus close monitoring and treatment of such patient should be provided at high dependency unit.

\section{References}

1. Mushtaq S, Singhi EK, Stuart RK (2015) “Triple-triple" hit lymphoma. Case Rep Intern Med 2: 78-80.

2. Swerdlow SH, Campo E, Pileri SA, Harris NL, Stein H, et al. (2016). The 2016 revision of the world health organization classification of lymphoid neoplasms. Blood 127: 2.375-2390.

3. Pemmaraju N, Gill J, Gupta S, Krause JR (2014) Triple-hit lymphoma. Proc (Bayl Univ Med Cent) 27: 125-127.

4. Chen D, Ketterling KP (2014) A leukemic presentation of a "triple-hit" lymphoma. Blood 123: 1126-1126.

5. Hashimoto A, Fujimi A, Kanisawa Y, Nakajima C, Hayasaka N, et al. (2015) Successful treatment with dose-adjusted EPOCH-R for triple-hit lymphoma having BCL2, BCL6 and MYC translocations. Rinsho Ketsueki 56: 905-910.

6. Wang W, Hu S, Lu X, Young KH, Medeiros LJ (2015) Triple-hit B-cell Lymphoma with MYC, BCL2, and BCL6 Translocations/Rearrangements, Clinicopathologic Features of 11 Cases. Am J Surg Pathol 39: 1132-1139.

7. Chang H, Lin TL, Ho WJ, Hsu LA (2008) Acute Myeloid Leukemia associated with acute myocardial infarction and dural sinus thrombosis: the possible role of leukemia-related hyperhomocysteinemia. J Chin Med Assoc 71: 416-420.

8. Jao GT, Knovich MA, Savage RW, Sane DC (2014) ST-elevation myocardial infarction and myelodysplastic syndrome with acute myeloid leukemia transformation. Tex Heart Inst J 41: 234-237.

9. Cohen Y, Amir G, Da'as N, Gillis S, Rund D, et al. (2002) Acute myocardial infarction as the presenting symptom of acute myeloblastic leukemia with extreme hyperleukocytosis. Am J of Hematology 71: 4749. 